\title{
Some new oscillation criteria for second-order hybrid differential equations
}

\author{
G. E. Chatzarakis ${ }^{1}$ (D), M. Deepa ${ }^{2}$ (D) N. Nagajothi ${ }^{3}$ (D), V. Sadhasivam³ (D) \\ ${ }^{1}$ School of Pedagogical and Technological Education (ASPETE), Department of Electrical and Electronic \\ Engineering Educators, N. Heraklio, Athens, 14121, Greece \\ ${ }^{2}$ Department of Mathematics, Pavai Arts and Science College for Women (Affli. to Periyar University), \\ Anaipalayam, Rasipuram - 637 401, Namakkal Dt. Tamil Nadu, India \\ ${ }^{3}$ Post Graduate and Research Department of Mathematics, Thiruvalluvar Government Arts College \\ (Affli. to Periyar University), Rasipuram - 637 401, Namakkal Dt. Tamil Nadu, India
}

\begin{abstract}
In this paper, we consider the second order hybrid differential equations. For this class of equations, we establish a new criterion to check whether all solutions of an equation, in this class, oscillate. We prove this criterion, using a generalized Riccati technique and an averaging method. The established oscillatory criteria have a distinct form, from all other relevant criteria, in the literature. We illustrate the validity of our results by means of various examples.
\end{abstract}

Mathematics Subject Classification (2010). 34A38, 34C10, 34K11

Keywords. Oscillation, hybrid differential equation, Riccati technique.

\section{Introduction}

The problem of oscillation or non-oscillation of the solutions of differential equations has been discussed by numerous authors and several techniques have been developed to deal with this problem. For the fundamental theory and preliminary results, we refer the reader to the books and articles in $[2,3,8,11,13,17,18,22,24]$. In the recent years, there has been much attention on various aspects of quadratic perturbations of nonlinear differential equations. The hybrid differential equation is an especially interesting type of nonlinear differential equations that is open to research. The reason is that hybrid differential equations include several dynamic systems, as special cases. There has been considerable work on the theory of hybrid differential equations. We refer the readers to the articles in $[7,9,20,23,27]$. Applications with numerical solutions have been studied by several authors, see for example, [10,14,16,25].

\footnotetext{
*Corresponding Author.

Email addresses: geaxatz@otenet.gr; gea.xatz@aspete.gr (G. E. Chatzarakis), mdeepa.maths@gmail.com (M. Deepa),nagajothi006@gmail.com (N. Nagajothi), ovsadha@gmail.com (V. Sadhasivam)

Received: 19.03.2019; Accepted: 12.09.2019
} 
In a review of the literature on hybrid differential equations, Dhage and Lakshmikantham [4] discussed the existence of extremal solutions and comparison result for first order hybrid differential equation with linear perturbations of the following type:

$$
\frac{d}{d t}\left(\frac{x(t)}{f(t, x(t))}\right)=g(t, x(t)) \text {, a.e. } t \in J, x\left(t_{0}\right)=x_{0} \in \mathbb{R},
$$

where $f \in C(J \times \mathbb{R}, \mathbb{R}-\{0\})$ and $g \in C(J \times \mathbb{R}, \mathbb{R})$.

On the other hand, there has been no work on the qualitative theory of hybrid differential equations. This has motivated us to extend the oscillation theory to hybrid differential equations of second order. In the present paper, we initiate the oscillation theory for hybrid differential equations of the form

$$
\left(\frac{x(t)}{f(t, x(t))}\right)^{\prime \prime}+q(t) x(t)=g(t, x(t)), t \geq t_{0} .
$$

Throughout this paper, we assume the following conditions hold:

$\left(A_{1}\right) q(t) \in C\left(\left[t_{0}, \infty\right), \mathbb{R}_{+}\right)$;

$\left(A_{2}\right) f(t, x(t)) \in C\left(\left[t_{0}, \infty\right) \times \mathbb{R}, \mathbb{R}_{+}\right)$, there exists a function $r(t) \in C^{\prime}\left(\left[t_{0}, \infty\right), \mathbb{R}_{+}\right)$such that $f(t, x(t)) \geq h(x) r(t) \geq M r(t)$, where $h(x)$ is not identically zero on $\left[t_{0}, \infty\right)$ and moreover, $|h(x)| \geq M>0$ and $\frac{d}{d t} f(t, x(t))>0$;

$\left(A_{3}\right) g(t, x(t)) \in C\left(\left[t_{0}, \infty\right) \times \mathbb{R}, \mathbb{R}\right)$, there exists a function $p(t) \in C\left(\left[t_{0}, \infty\right), \mathbb{R}_{+}\right)$such that $\frac{g(t, x(t))}{x(t)} \leq p(t)$ for $x \neq 0, t \geq t_{0}$ and $q(t) \geq p(t)$.

Note that if $f(t, x(t))=1, g(t, x(t))=0$, then equation (1.1) is reduced to the linear differential equations of second order

$$
x^{\prime \prime}(t)+q(t) x(t)=0, t \geq t_{0},
$$

which include several equations, namely, the famous Euler equation that has been studied by many authors $[1,5,6,12,15,19,21,26]$.

By a solution of (1.1), we mean a nontrivial function $x(t) \in C^{2}\left(\left[T_{0}, \infty\right)\right), T_{0} \geq t_{0}$ which satisfies (1.1) on $\left[T_{0}, \infty\right)$. We only consider those solutions $x(t)$ of (1.1) satisfying $\sup \{|x(t)|: t \geq T\}>0$ for all $T \geq T_{0}$, and we assume that (1.1) possesses such solutions. A solution of (1.1) is called oscillatory if it has arbitrarily many zeros on $\left[t_{0}, \infty\right)$, and is called nonoscillatory otherwise. Equation (1.1) is said to be oscillatory if all its solutions are oscillatory.

The aim in this paper is to present some new oscillation criteria for (1.1) by using generalized Riccati technique and an integral averaging method. The contribution is original, as no results on the oscillation of nonlinear hybrid differential equations having been reported in the literature.

The paper is divided in three sections. In Section 2, we establish some new oscillation criteria for (1.1) while in the final section, we present some examples to illustrate the effectiveness of our main results.

\section{Main results}

In this section, we present sufficient conditions, which guarantee the oscillatory behavior of the solutions of equation (1.1). We begin with the following theorem.

Theorem 2.1. Suppose that the assumptions $\left(A_{1}\right)-\left(A_{3}\right)$ hold. Moreover, assume that there exists a positive nondecreasing function $\delta \in C^{1}\left(\left[t_{0}, \infty\right) ;(0, \infty)\right)$ such that for all sufficiently large $t_{1} \geq t_{0}$, we have

$$
\limsup _{t \rightarrow \infty} \int_{t_{1}}^{t}\left(\delta(s)(q(s)-p(s))-\frac{1}{4 M} \frac{\left(\delta^{\prime}(s)\right)^{2}}{r(s) \delta(s)}\right) d s=\infty .
$$

Then every solution $x(t)$ of (1.1) is oscillatory. 
Proof. Suppose that $x(t)$ is a nonoscillatory solution of (1.1). Without loss of generality we may assume that $x(t)>0$ for $t \geq t_{1} \geq t_{0}$, since similar arguments can be made, for the case $x(t)<0$, eventually. Now, from (1.1), we have

$$
\left(\frac{x(t)}{f(t, x(t))}\right)^{\prime \prime} \leq 0 \text { for } t \geq t_{1} \text {. }
$$

Therefore $\left(\frac{x(t)}{f(t, x(t))}\right)^{\prime}$ is a decreasing function. We now claim that $\left(\frac{x(t)}{f(t, x(t))}\right)^{\prime}>0$ for $t \geq t_{1}$. If not, then there exists $t_{2} \geq t_{1}$ such that

$$
\left(\frac{x(t)}{f(t, x(t))}\right)^{\prime} \leq\left.\left(\frac{x(t)}{f(t, x(t))}\right)^{\prime}\right|_{t=t_{2}}:=c<0, t \geq t_{2} .
$$

Integrating from $t_{2}$ to $t$, we get

$$
x(t) \leq\left(c\left(t-t_{2}\right)+d\right) f(t, x(t)) \rightarrow-\infty \text { as } t \rightarrow \infty,
$$

where $d=\frac{x\left(t_{2}\right)}{f\left(t_{2}, x\left(t_{2}\right)\right)}$, which contradicts the fact that $x(t)>0$ for $t \geq t_{1}$.

Define the function $w(t)$ by the generalized Riccati substitution

$$
w(t)=\delta(t)\left(\frac{x(t)}{f(t, x(t))}\right)^{\prime} \frac{1}{x(t)}, t \geq t_{1} .
$$

Then $w(t)>0$ for $t \geq t_{1}$. Differentiating (2.3) and using (1.1) and $\left(A_{3}\right)$, we have

$$
\begin{aligned}
w^{\prime}(t) & =\frac{\delta^{\prime}(t)}{\delta(t)} w(t)+\frac{\delta(t)}{x(t)}(g(t, x(t))-q(t) x(t))-w(t) \frac{x^{\prime}(t)}{x(t)} \\
& \leq \frac{\delta^{\prime}(t)}{\delta(t)} w(t)+\delta(t) p(t)-q(t) \delta(t)-w^{2}(t) \frac{x^{\prime}(t)}{\delta(t)\left(\frac{x(t)}{f(t, x(t))}\right)^{\prime}} .
\end{aligned}
$$

By $\left(A_{2}\right)$, the last inequality becomes

$$
\begin{aligned}
w^{\prime}(t) & \leq \frac{\delta^{\prime}(t)}{\delta(t)} w(t)+\delta(t)(p(t)-q(t))-\frac{w^{2}(t)}{\delta(t)} f(t, x(t)) \\
& \leq \frac{\delta^{\prime}(t)}{\delta(t)} w(t)-\delta(t)(q(t)-p(t))-M \frac{r(t)}{\delta(t)} w^{2}(t) .
\end{aligned}
$$

Using the inequality, $B u-A u^{2} \leq \frac{B^{2}}{4 A}$, we have

$$
w^{\prime}(t) \leq-\delta(t)(q(t)-p(t))+\frac{1}{4 M} \frac{\left(\delta^{\prime}(t)\right)^{2}}{r(t) \delta(t)} .
$$

Integrating the last inequality from $t_{1}$ to $t$ and taking the limit supremum on both sides, yields

$$
\limsup _{t \rightarrow \infty} \int_{t_{1}}^{t}\left(\delta(s)(q(s)-p(s))-\frac{1}{4 M} \frac{\left(\delta^{\prime}(s)\right)^{2}}{r(s) \delta(s)}\right) d s \leq w\left(t_{1}\right), t \geq t_{1},
$$

which contradicts hypothesis (2.1). The proof of the theorem is complete.

Next we present some new oscillation results for (1.1).

We introduce the class of functions $\Omega$. Let $D=\left\{(t, s): t_{0} \leq s \leq t\right\}$. The function $H \in$ $C(D, \mathbb{R})$ is said to belong to the class $\Omega$, if

$\left(T_{1}\right) H(t, t)=0$ for $t \geq t_{0}$ and $H(t, s)>0$ for $t>s \geq t_{0}$.

$\left(T_{2}\right) H$ has continuous and nonpositive partial derivatives on $D$ with respect to s and there exists a function $h_{1}(t, s) \in C(D, \mathbb{R})$ such that 
(i) $h_{1}(t, s) \sqrt{H(t, s)}=-\frac{\partial H}{\partial s}(t, s)$,

(ii) $h_{2}(t, s)=h_{1}(t, s)-\sqrt{H(t, s)} \frac{\delta^{\prime}(s)}{\delta(s)}$.

Theorem 2.2. Assume that $\left(A_{1}\right)-\left(A_{3}\right)$ hold. In addition, assume that there exists a positive function $\delta \in C^{1}\left(\left[t_{0}, \infty\right) ;(0, \infty)\right)$ such that for all sufficiently large $t_{1} \geq t_{0}$, we have

$$
\limsup _{t \rightarrow \infty} \frac{1}{H\left(t, t_{0}\right)} \int_{t_{0}}^{t}\left(H(t, s) \delta(s)(q(s)-p(s))-\frac{1}{4 M} \frac{h_{2}^{2}(t, s) \delta(s)}{r(s)}\right) d s=\infty .
$$

Then every solution $x(t)$ of (1.1) is oscillatory.

Proof. Suppose that $x(t)$ is a nonoscillatory solution of (1.1). Without loss of generality we may assume that $x(t)>0$ for $t \geq t_{1}$ for some $t_{1} \geq t_{0}$. Multiplying both sides of $(2.4)$ by $H(t, s)$, integrating it with respect to $\mathrm{s}$ from $t_{1}$ to $t$ and using the properties of the function $H(t, s)$ for all $t \geq t_{1} \geq t_{0}$, we get

$$
\begin{aligned}
& \int_{t_{1}}^{t} H(t, s) \delta(s)(q(s)-p(s)) d s \\
& \leq-\int_{t_{1}}^{t} H(t, s) w^{\prime}(s) d s+\int_{t_{1}}^{t} H(t, s) \frac{\delta^{\prime}(s)}{\delta(s)} w(s) d s-\int_{t_{1}}^{t} M H(t, s) \frac{r(s)}{\delta(s)} w^{2}(s) d s \\
& \leq H\left(t, t_{1}\right) w\left(t_{1}\right)-\int_{t_{1}}^{t} h_{2}(t, s) \sqrt{H(t, s)} w(s) d s-\int_{t_{1}}^{t} M H(t, s) \frac{r(s)}{\delta(s)} w^{2}(s) d s \\
& \leq H\left(t, t_{1}\right) w\left(t_{1}\right)-\int_{t_{1}}^{t}\left(\sqrt{M H(t, s) \frac{r(s)}{\delta(s)}} w(s)+\frac{1}{2} \frac{h_{2}(t, s) \sqrt{\delta(s)}}{\sqrt{M r(s)}}\right)^{2} d s \\
& \quad+\int_{t_{1}}^{t} \frac{1}{4 M} \frac{\delta(s)}{r(s)} h_{2}^{2}(t, s) d s .
\end{aligned}
$$

Thus, we conclude that for every $t \geq t_{0}$,

$$
\begin{aligned}
& \int_{t_{1}}^{t}\left(H(t, s) \delta(s)(q(s)-p(s))-\frac{1}{4 M} \frac{\delta(s)}{r(s)} h_{2}^{2}(t, s)\right) d s \\
& \quad \leq H\left(t, t_{1}\right) w\left(t_{1}\right)-\int_{t_{1}}^{t}\left(\sqrt{M H(t, s) \frac{r(s)}{\delta(s)}} w(s)+\frac{1}{2} \frac{h_{2}(t, s) \sqrt{\delta(s)}}{\sqrt{M r(s)}}\right)^{2} d s \\
& \quad \leq H\left(t, t_{1}\right) w\left(t_{1}\right) \leq H\left(t, t_{0}\right)\left|w\left(t_{0}\right)\right|,
\end{aligned}
$$

which implies that

$$
\begin{gathered}
\int_{t_{0}}^{t}\left(H(t, s) \delta(s)(q(s)-p(s))-\frac{1}{4 M} \frac{\delta(s)}{r(s)} h_{2}^{2}(t, s)\right) d s \\
\quad \leq H\left(t, t_{0}\right)\left(\int_{t_{0}}^{t_{1}}(\delta(s)(q(s)-p(s))) d s+\left|w\left(t_{0}\right)\right|\right) .
\end{gathered}
$$


Inequality (2.7) yields

$$
\begin{gathered}
\limsup _{t \rightarrow \infty} \frac{1}{H\left(t, t_{0}\right)} \int_{t_{0}}^{t}\left(H(t, s) \delta(s)(q(s)-p(s))-\frac{1}{4 M} \frac{\delta(s)}{r(s)} h_{2}^{2}(t, s)\right) d s \\
\leq \int_{t_{0}}^{t_{1}}(\delta(s)(q(s)-p(s))) d s+\left|w\left(t_{0}\right)\right|<\infty .
\end{gathered}
$$

which contradicts $(2.5)$. The proof of the theorem is complete.

The following corollaries can easily be derived, from Theorem 2.2.

Corollary 2.3. Assume that the conditions of Theorem 2.2 hold with (2.5) replaced by

$$
\limsup _{t \rightarrow \infty} \frac{1}{H\left(t, t_{0}\right)} \int_{t_{0}}^{t}(H(t, s) \delta(s)(q(s)-p(s))) d s=\infty
$$

and

$$
\limsup _{t \rightarrow \infty} \frac{1}{H\left(t, t_{0}\right)} \int_{t_{0}}^{t} \frac{1}{4 M} \frac{\delta(s)}{r(s)} h_{2}^{2}(t, s) d s<\infty .
$$

Then every solution $x(t)$ of (1.1) is oscillatory.

Theorem 2.2 enables us to derive many sufficient conditions for (1.1) with different choices of the function $H$.

Consider $H(t, s)=(t-s)^{n-1},(t, s) \in D$ for some integer $n>2$. Then, Theorem 2.2 leads to the following result.

Corollary 2.4. Assume that the conditions of Theorem 2.2 hold, equation (2.5) can be written as

$$
\begin{aligned}
\limsup _{t \rightarrow \infty} \frac{1}{\left(t-t_{0}\right)^{n-1}} \int_{t_{0}}^{t} & \left((t-s)^{n-1} \delta(s)((q(s)-p(s))\right. \\
& \left.\left.-\frac{1}{4 M r(s)}\left(\frac{\delta^{\prime}(s)}{\delta(s)}-\frac{n-1}{t-s}\right)^{2}\right)\right) d s=\infty
\end{aligned}
$$

for some integer $n>2$. Then every solution $x(t)$ of (1.1) is oscillatory.

Let $H(t, s)=(R(t)-R(s))^{\lambda}$, where $\lambda$ is a constant, $R(t)=\int_{t_{1}}^{t} \frac{1}{r(s)} d s$ and $\lim _{t \rightarrow \infty} R(t)=$ $\infty$. Then, Theorem 2.2 implies the following result.

Corollary 2.5. Assume that the conditions of Theorem 2.2 hold, equation (2.5) can be written as

$$
\begin{aligned}
\limsup _{t \rightarrow \infty} \frac{1}{\left(R(t)-R\left(t_{0}\right)\right)^{\lambda}} & \int_{t_{0}}^{t}\left((R(t)-R(s))^{\lambda} \delta(s)((q(s)-p(s))\right. \\
& \left.\left.-\frac{1}{4 M r(s)}\left(\frac{\delta^{\prime}(s)}{\delta(s)}-\frac{\lambda}{r(s)(R(t)-R(s))}\right)^{2}\right)\right) d s=\infty .
\end{aligned}
$$

Then every solution $x(t)$ of (1.1) is oscillatory.

Let $H(t, s)=\left(\log \left(\frac{t}{s}\right)\right)^{n}, t>s>t_{1}, n>1$ is an integer. Then, from Theorem 2.2, we get the following result. 
Corollary 2.6. Assume that the conditions of Theorem 2.2 hold, equation (2.5) can be written as

$$
\begin{aligned}
\limsup _{t \rightarrow \infty} \frac{1}{\left(\log \left(\frac{t}{t_{0}}\right)\right)^{n}} & \int_{t_{0}}^{t}\left(\left(\log \left(\frac{t}{s}\right)\right)^{n} \delta(s)((q(s)-p(s))\right. \\
& \left.\left.-\frac{1}{4 M r(s)}\left(\frac{\delta^{\prime}(s)}{\delta(s)}-\frac{n}{s\left(\log \left(\frac{t}{s}\right)\right)}\right)^{2}\right)\right) d s=\infty
\end{aligned}
$$

Then every solution $x(t)$ of (1.1) is oscillatory.

Let $H(t, s)=\left(\int_{s}^{t} \frac{d u}{\theta(u)}\right)^{n}, t>s>t_{0}$, where $n>1$ is an integer and $\theta:\left[t_{0}, \infty\right) \rightarrow \mathbb{R}_{+}$ is a continuous function such that $\lim _{t \rightarrow \infty}\left(\int_{t_{0}}^{t} \frac{d u}{\theta(u)}\right)=\infty$. Then Theorem 2.2 yields the following result.

Corollary 2.7. Assume that the conditions of Theorem 2.2 hold, equation (2.5) can be written as

$$
\begin{aligned}
\limsup _{t \rightarrow \infty}\left(\int_{t_{0}}^{t} \frac{d u}{\theta(u)}\right)^{-n} & \int_{t_{0}}^{t}\left(\left(\int_{s}^{t} \frac{d u}{\theta(u)}\right)^{n} \delta(s)((q(s)-p(s))\right. \\
& \left.\left.-\frac{1}{4 M r(s)}\left(\frac{\delta^{\prime}(s)}{\delta(s)}-\frac{n}{\theta(s)\left(\int_{s}^{t} \frac{d u}{\theta(u)}\right)}\right)^{2}\right)\right) d s=\infty .
\end{aligned}
$$

Then every solution $x(t)$ of (1.1) is oscillatory.

Theorem 2.8. Assume that

$$
0<\inf _{s \geq t_{0}}\left(\liminf _{t \rightarrow \infty} \frac{H(t, s)}{H\left(t, t_{0}\right)}\right) \leq \infty
$$

and

$$
\limsup _{t \rightarrow \infty} \frac{1}{H\left(t, t_{0}\right)} \int_{t_{0}}^{t} \frac{\delta(s)}{r(s)} h_{2}^{2}(t, s) d s<\infty .
$$

Then (1.1) is oscillatory if there exists a continuous function $\psi$ on $\left[t_{0}, \infty\right)$ with

$$
\int_{t_{0}}^{\infty} \frac{r(s)}{\delta(s)} \psi_{+}^{2}(s) d s=\infty
$$

where $\psi_{+}(t)=\max \{\psi(t), 0\}$ and such that

$$
\limsup _{t \rightarrow \infty} \frac{1}{H(t, T)} \int_{T}^{t}\left(H(t, s) \delta(s)(q(s)-p(s))-\frac{1}{4 M} \frac{\delta(s)}{r(s)} h_{2}^{2}(t, s)\right) d s \geq \psi(T),
$$

for every $T \geq t_{0}$.

Proof. Let $x(t)$ be a nonoscillatory solution of (1.1). Then there exists a $T_{0} \geq t_{0}$ such that $x(t) \neq 0$ for all $t \geq T_{0}$. We define $w(t)$ as in $(2.3)$ on $\left[T_{0}, \infty\right)$. As in the proof of Theorem 2.2, inequality (2.6) is satisfied for all $t$, $t_{1}$ with $t \geq t_{1}=T \geq T_{0}$. So, for $t \geq T \geq T_{0}$, we obtain the inequality

$$
\begin{aligned}
& \frac{1}{H(t, T)} \int_{T}^{t}\left(H(t, s) \delta(s)(q(s)-p(s))-\frac{1}{4 M} \frac{\delta(s)}{r(s)} h_{2}^{2}(t, s)\right) d s \\
& \leq w(T)-\frac{1}{H(t, T)} \int_{T}^{t}\left(\sqrt{M H(t, s) \frac{r(s)}{\delta(s)}} w(s)+\frac{1}{2} \frac{h_{2}(t, s) \sqrt{\delta(s)}}{\sqrt{M r(s)}}\right)^{2} d s
\end{aligned}
$$


and therefore

$$
\begin{aligned}
& \limsup _{t \rightarrow \infty} \frac{1}{H(t, T)} \int_{T}^{t}\left(H(t, s) \delta(s)(q(s)-p(s))-\frac{1}{4 M} \frac{\delta(s)}{r(s)} h_{2}^{2}(t, s)\right) d s \\
& \leq w(T)-\liminf _{t \rightarrow \infty} \frac{1}{H(t, T)} \int_{T}^{t}\left(\sqrt{M H(t, s) \frac{r(s)}{\delta(s)}} w(s)+\frac{1}{2} \frac{h_{2}(t, s) \sqrt{\delta(s)}}{\sqrt{M r(s)}}\right)^{2} d s .
\end{aligned}
$$

Thus, by (2.17), we get

$$
w(T) \geq \psi(T)+\liminf _{t \rightarrow \infty} \frac{1}{H(t, T)} \int_{T}^{t}\left(\sqrt{M H(t, s) \frac{r(s)}{\delta(s)}} w(s)+\frac{1}{2} \frac{h_{2}(t, s) \sqrt{\delta(s)}}{\sqrt{M r(s)}}\right)^{2} d s,
$$

for all $t>T \geq T_{0}$. This implies that

$$
w(T) \geq \psi(T) \text { for all } T \geq T_{0},
$$

and

$$
\begin{aligned}
\liminf _{t \rightarrow \infty} \frac{1}{H(t, T)} \int_{T}^{t}\left(\sqrt{M H(t, s) \frac{r(s)}{\delta(s)}} w(s)\right. & \left.+\frac{1}{2} \frac{h_{2}(t, s) \sqrt{\delta(s)}}{\sqrt{M r(s)}}\right)^{2} d s \\
& \leq w\left(T_{0}\right)-\psi\left(T_{0}\right)<\infty
\end{aligned}
$$

that is,

$$
\liminf _{t \rightarrow \infty}(\theta(t)+\eta(t))<\infty,
$$

where

$$
\begin{aligned}
& \theta(t)=\frac{1}{H\left(t, T_{0}\right)} \int_{T_{0}}^{t} M \frac{r(s)}{\delta(s)} H(t, s) w^{2}(s) d s, t>T_{0}, \\
& \eta(t)=\frac{1}{H\left(t, T_{0}\right)} \int_{T_{0}}^{t} \sqrt{H(t, s)} h_{2}(t, s) w(s) d s, t>T_{0} .
\end{aligned}
$$

In order to show that

$$
\int_{T_{0}}^{t} \frac{r(s)}{\delta(s)} w^{2}(s) d s<\infty
$$

suppose that

$$
\int_{T_{0}}^{t} \frac{r(s)}{\delta(s)} w^{2}(s) d s=\infty
$$

Indeed, let $\alpha$ be a positive constant. Then, by condition (2.14), we get

$$
\inf _{s \geq t_{0}}\left(\liminf _{t \rightarrow \infty} \frac{H(t, s)}{H\left(t, t_{0}\right)}\right)>\alpha>0 .
$$

On the other hand, for any positive constant $\beta$, due to (2.24), there exists a $T_{1}>T_{0}$ such that

$$
\int_{T_{0}}^{t} \frac{r(s)}{\delta(s)} w^{2}(s) d s \geq \frac{\beta}{\alpha} \text { for all } t \geq T_{1}
$$


Therefore

$$
\begin{aligned}
\theta(t) & =\frac{M}{H\left(t, T_{0}\right)} \int_{T_{0}}^{t} H(t, s) d\left(\int_{T_{0}}^{s} \frac{r(u)}{\delta(u)} w^{2}(u) d u\right) \\
& =\frac{M}{H\left(t, T_{0}\right)} \int_{T_{0}}^{t}\left(\int_{T_{0}}^{s} \frac{r(u)}{\delta(u)} w^{2}(u) d u\right)\left(-\frac{\partial H(t, s)}{\partial s}\right) d s \\
& \geq \frac{M \beta}{\alpha H\left(t, T_{0}\right)} \int_{T_{1}}^{t}\left(-\frac{\partial H(t, s)}{\partial s}\right) d s=\frac{M \beta}{\alpha} \frac{H\left(t, T_{1}\right)}{H\left(t, T_{0}\right)} .
\end{aligned}
$$

But

$$
\frac{H\left(t, T_{1}\right)}{H\left(t, t_{0}\right)}>\alpha
$$

then there exists $T_{2} \geq T_{1}$ such that $\liminf _{t \rightarrow \infty} \frac{H\left(t, T_{1}\right)}{H\left(t, t_{0}\right)} \geq \alpha$, for all $t \geq T_{2}$. So, we have $\theta(t) \geq M \beta$ for all $t \geq T_{2}$. Then,

$$
\lim _{t \rightarrow \infty} \theta(t)=\infty
$$

since $\beta$ is an arbitrary positive constant.

Next, let us consider a sequence $\left\{\tau_{l}\right\}_{l=1,2, \ldots}$ in $\left(T_{0}, \infty\right)$ with $\tau_{l} \rightarrow \infty$ as $l \rightarrow \infty$ and such that

$$
\lim _{l \rightarrow \infty}\left(\theta\left(\tau_{l}\right)-\eta\left(\tau_{l}\right)\right)=\liminf _{t \rightarrow \infty}(\theta(t)-\eta(t))
$$

Now, by (2.20), there exists a constant $\mu$ such that

$$
\theta\left(\tau_{l}\right)-\eta\left(\tau_{l}\right) \leq \mu(l=1,2, \ldots) .
$$

Furthermore, (2.26) guarantees that

$$
\lim _{l \rightarrow \infty} \theta\left(\tau_{l}\right)=\infty
$$

and hence (2.27) gives

$$
\lim _{l \rightarrow \infty} \eta\left(\tau_{l}\right)=-\infty .
$$

Taking into account (2.28) and using (2.27), we derive

$$
1+\frac{\eta\left(\tau_{l}\right)}{\theta\left(\tau_{l}\right)} \leq \frac{\mu}{\theta\left(\tau_{l}\right)}<k, 0<k<1 \text { for large } l .
$$

From (2.29), this implies that

$$
\begin{aligned}
\frac{\eta^{2}\left(\tau_{l}\right)}{\theta\left(\tau_{l}\right)} & >(k-1) \eta\left(\tau_{l}\right), \\
\lim _{l \rightarrow \infty} \frac{\eta^{2}\left(\tau_{l}\right)}{\theta\left(\tau_{l}\right)} & =\infty
\end{aligned}
$$


By Schwarz's inequality, we have

$$
\begin{aligned}
\eta^{2}\left(\tau_{l}\right) & =\frac{1}{H^{2}\left(\tau_{l}, T_{0}\right)}\left(\int_{T_{0}}^{\tau_{l}} \sqrt{H\left(\tau_{l}, s\right)} h_{2}^{2}\left(\tau_{l}, s\right) w(s) d s\right)^{2} \\
& \leq\left(\frac{1}{H\left(\tau_{l}, T_{0}\right)} \int_{T_{0}}^{\tau_{l}} \frac{\delta(s)}{r(s)} h_{2}^{2}\left(\tau_{l}, s\right) d s\right)\left(\frac{1}{H\left(\tau_{l}, T_{0}\right)} \int_{T_{0}}^{\tau_{l}} H\left(\tau_{l}, s\right) \frac{r(s)}{\delta(s)} w^{2}(s) d s\right) \\
& \leq \frac{\theta\left(\tau_{l}\right)}{M}\left(\frac{1}{H\left(\tau_{l}, T_{0}\right)} \int_{T_{0}}^{\tau_{l}} \frac{\delta(s)}{r(s)} h_{2}^{2}\left(\tau_{l}, s\right) d s\right),
\end{aligned}
$$

and therefore

$$
\frac{\eta^{2}\left(\tau_{l}\right)}{\theta\left(\tau_{l}\right)} \leq \frac{1}{M \alpha}\left(\frac{1}{H\left(\tau_{l}, t_{0}\right)} \int_{t_{0}}^{\tau_{l}} \frac{\delta(s)}{r(s)} h_{2}^{2}\left(\tau_{l}, s\right) d s\right) .
$$

Using (2.31), we have

$$
\lim _{l \rightarrow \infty} \frac{1}{H\left(\tau_{l}, t_{0}\right)} \int_{t_{0}}^{\tau_{l}} \frac{\delta(s)}{r(s)} h_{2}^{2}\left(\tau_{l}, s\right) d s=\infty
$$

which gives

$$
\limsup _{l \rightarrow \infty} \frac{1}{H\left(t, t_{0}\right)} \int_{t_{0}}^{t} \frac{\delta(s)}{r(s)} h_{2}^{2}(t, s) d s=\infty,
$$

which contradicts (2.15). Hence (2.24) fails to hold. Finally,

$$
\int_{t_{0}}^{\infty} \frac{r(s)}{\delta(s)} \psi_{+}^{2}(s) d s \leq \int_{t_{0}}^{\infty} \frac{r(s)}{\delta(s)} w^{2}(s) d s<\infty
$$

which contradicts (2.16). Therefore (1.1) is oscillatory.

Theorem 2.9. Let all the assumptions of Theorem 2.8 hold, except for (2.15) which is replaced by

$$
\limsup _{t \rightarrow \infty} \frac{1}{H\left(t, t_{0}\right)} \int_{t_{0}}^{t} H(t, s) \delta(s)(q(s)-p(s)) d s<\infty .
$$

Then every solution of (1.1) is oscillatory.

The proof is similar to that of Theorem 2.8, and hence is omitted.

We conclude with the following remarks that suggest some open problems, for future research.

Remark 2.10. The results obtained in this article can be extended for the more general differential equation having a damped term

$$
\left(\frac{x(t)}{f(t, x(t))}\right)^{\prime \prime}+p(t)\left(\frac{x(t)}{f(t, x(t))}\right)^{\prime}+q(t) h(x(t))=g(t, x(t)), t \geq t_{0},
$$

where $p(t)$ is a continuous function on $\left[t_{0}, \infty\right)$.

Remark 2.11. All above results can be extended to the following form

$$
\left(b(t)\left(a(t) \frac{x(t)}{f(t, x(t))}\right)^{\prime}\right)^{\prime}+q(t) h(x(t))=g(t, x(t)), t \geq t_{0} .
$$


Remark 2.12. The results obtained in this paper merely initiate the study of the oscillations of hybrid differential equations. Therefore, this problem remains largely open, for future research.

\section{Examples}

In this section, we provide some examples to illustrate our main results.

Example 3.1. Consider the hybrid differential equation

$$
\left(\frac{x(t)}{e^{t}}\right)^{\prime \prime}+x(t)=\left(e^{t}-1\right) \sin t, \quad t \geq t_{0}
$$

Here $q(t)=1, M=1, f(t, x(t))=e^{t}, r(t)=1, g(t, x(t))=\left(e^{t}-1\right) \sin t$ and $\frac{g(t, x(t))}{x(t)}=1-\frac{1}{e^{t}}$. Now, choose $\epsilon>0$ such that $1-\frac{1}{e^{t}}<\epsilon<1$ and $p(t)=\epsilon$ with $\delta(t)=1$. Consider

$$
\begin{aligned}
& \limsup _{t \rightarrow \infty} \int_{t_{1}}^{t}\left(\delta(s)(q(s)-p(s))-\frac{1}{4 M} \frac{\left(\delta^{\prime}(s)\right)^{2}}{r(s) \delta(s)}\right) d s \\
&=\limsup _{t \rightarrow \infty} \int_{t_{1}}^{t}(1-\epsilon) d s \rightarrow \infty \text { as } t \rightarrow \infty .
\end{aligned}
$$

Hence, all the conditions of Theorem 2.1 are satisfied. Therefore, every solution of (3.1) is oscillatory. In fact, $x(t)=e^{t} \sin t$ is one such solution of (3.1).

Example 3.2. Consider the second-order hybrid differential equation

$$
\left(\frac{x(t)}{t}\right)^{\prime \prime}+\frac{1}{t^{2}} x(t)=(t+3) e^{t}, \quad t \geq 1
$$

Here $q(t)=\frac{1}{t^{2}}, g(t, x(t))=(t+3) e^{t}, M=1, f(t, x(t))=t, r(t)=1$ and $p(t)=\frac{t+4}{t^{2}}$ with $\delta(t)=1$. Consider

$$
\begin{aligned}
\limsup _{t \rightarrow \infty} & \int_{t_{1}}^{t}\left(\delta(s)(q(s)-p(s))-\frac{1}{4 M} \frac{\left(\delta^{\prime}(s)\right)^{2}}{r(s) \delta(s)}\right) d s \\
& =\limsup _{t \rightarrow \infty} \int_{t_{1}}^{t}\left(\frac{1}{s^{2}}-\frac{s+4}{s^{3}}\right) d s \leq \limsup _{t \rightarrow \infty} \int_{t_{1}}^{t} \frac{1}{s^{2}} d s=\infty .
\end{aligned}
$$

Thus, the conditions of Theorem 2.1 are not satisfied. In fact, $x(t)=t^{2} e^{t}$ is a nonoscillatory solution of (3.2).

Example 3.3. Consider the hybrid differential equation of second order

$$
\left(\frac{x(t)}{e^{t}}\right)^{\prime \prime}+x(t)=\left(e^{t}-1\right) \sin t, \quad t \geq t_{0} .
$$

Here $q(t)=1, M=1, f(t, x(t))=e^{t}, r(t)=1, g(t, x(t))=\left(e^{t}-1\right) \sin t$ and $\frac{g(t, x(t))}{x(t)}=1-\frac{1}{e^{t}}$. Now, choose $\epsilon>0$ such that $1-\frac{1}{e^{t}}<\epsilon<1, p(t)=\epsilon$ and $\delta(t)=1$ with $H(t, s)=t-s$ and 


$$
\begin{aligned}
& h_{1}(t, s)=h_{2}(t, s)=\frac{1}{\sqrt{t-s}} \text {. Consider } \\
& \qquad \begin{aligned}
\limsup _{t \rightarrow \infty} & \frac{1}{H\left(t, t_{0}\right)} \int_{t_{0}}^{t}\left(H(t, s) \delta(s)(q(s)-p(s))-\frac{1}{4 M} \frac{h_{2}^{2}(t, s) \delta(s)}{r(s)}\right) d s \\
& =\limsup _{t \rightarrow \infty} \frac{1}{\left(t-t_{0}\right)} \int_{t_{0}}^{t}\left((t-s)(1-\epsilon)-\frac{1}{4} \frac{1}{t-s}\right) d s \rightarrow \infty \text { as } t \rightarrow \infty .
\end{aligned}
\end{aligned}
$$

Thus, all the conditions of Theorem 2.2 are satisfied. Hence every solution of (3.3) is oscillatory. For example, $x(t)=e^{t} \cos t$ is one such solution.

Acknowledgment. The authors would like to thank the anonymous reviewers for their valuable comments and suggestions to improve the quality of the manuscript.

\section{References}

[1] H.Kh. Abdullah, Oscillation conditions of second-order nonlinear differential equations, Int. J. Math. Sci. 34 (1), 1490-1497, 2014.

[2] R.P. Agarwal, S.R. Grace and D. O'Regan, Oscillation theory for difference and differential equations, Kluwer academic publishers, Dordrecht, 2000.

[3] R.P. Agarwal and D. O'Regan, An introduction to ordinary differential equations, Springer, Newyork, 2008.

[4] B.C. Dhage and V. Lakshmikantham, Basic results on hybrid differential equations, Nonlinear Anal. Hybrid Syst. 4, 414-424, 2010.

[5] E.M. Elabbasy, T.S. Hassan and S.H. Saker, Oscillation of second-order nonlinear differential equations with a damping term, Electron. J. Differ. Eq. 76, 1-13, 2005.

[6] X. Fu, T. Li and C. Zhang, Oscillation of second-order damped differential equations, Adv. Difference Equ. 326, 2013.

[7] H. Ge and J. Xin, On the existence of a mild solution for impulsive hybrid fractional differential equations, Adv. Difference Equ. 211, 2013.

[8] S.R. Grace, Oscillation theorems for nonlinear differential equations of second order, J. Math. Anal. Appl. 171, 220-241, 1992.

[9] M.A.E. Herzallah and D. Baleanu, On fractional order hybrid differential equations, Abstr. Appl. Anal. 2014, Article ID 389386, 2014.

[10] M. Heydari, G.B. Loghmani, S.M. Hosseini and S.M. Karbassi, Application of hybrid functions for solving diffing-harmonic oscillator, J. Difference Equ. 2014, Article ID 210754, 2014.

[11] W.G. Kelley and A.C. Peterson, The theory of differential equations: classical and qualitative, Springer, NewYork, 2010.

[12] H. Liu and F. Meng, Interval oscillation criteria for second-order nonlinear forced differential equations involving variable exponent, Adv. Difference Equ. 291, 2016.

[13] Z. Luo and J. Shen, Oscillation of second order linear differential equations with impulses, Appl. Math. Lett. 20, 75-81, 2007.

[14] K. Maleknejad and L. Torkzadeh, Application of hybrid functions for solving oscillator equations, Rom. Journ. Phys. 60 (1-2), 87-98, 2015.

[15] J.V. Manojlovic, Oscillation criteria for second-order half-linear differential equations, Math. Comput. Modelling 30, 109-119, 1999.

[16] P. Micheau and P. Coirault, A harmonic controller of engine speed oscillations for hybrid vehicles, Elsevier IFAC publications, 19-24, 2005.

[17] A.K. Nandakumaran, P.S. Datti and R.K. George, Ordinary differential equations, principles and applications, Cambridge University Press, 2017. 
[18] A. Ozbekler, J.S.W. Wong, A. Safer, Forced oscillation of second-order nonlinear differential equations with positive and negative coefficients, Appl. Math. Lett. 24, 1225-1230, 2011.

[19] Ch.G. Philos, Oscillation theorems for linear differential equations of second order, Arch. Math. 53, 482-492, 1989.

[20] V. Sadhasivam and M. Deepa, Oscillation criteria for fractional impulsive hybrid partial differential equations, Probl. Anal. Issues Anal. 8 (26), 73-91, 2019.

[21] S.H. Saker, Oscillation theory of delay differential and difference equations, VDM Verlag, Dr.Muller Aktiengesellschaft and Co, USA, 2010.

[22] S.H. Saker, P.Y.H. Pang and R.P. Agarwal, Oscillation theorems for second order nonlinear functional differential equations, Dynam. Systems Appl. 12, 307-322, 2003.

[23] S. Sitho, S.K. Ntouyas and J. Tariboon, Existence results for hybrid fractional integrodifferential equations, Bound. Value Probl. 113, 1-13, 2015.

[24] J.S.W. Wong, On Kamenev-type oscillation Theorems for second-order differential equations with damping, J. Math. Anal. Appl. 258, 244-257, 2001.

[25] F. Yuan and D. DiClemente, Hybrid voltage-controlled oscillator with low phase noise andlarge frequency tunig range, Analog Integr Circ Sig Process 82, 471-478, 2015.

[26] Y. Zhao, S. Sun, Z. Han and Q. Li, Theory of fractional hybrid differential equations, Comput. Math. Appl. 62, 1312-1324, 2011.

[27] A. Zhao, Y. Wang and J. Yan, Oscillation criteria for second-order nonlinear differential equations with nonlinear damping, Comput. Math. Appl. 56, 542-555, 2008. 